\title{
Anatomy of nuclear shape transition in the relativistic mean field theory
}

\author{
Tapas Sil ${ }^{1}$, B. K. Agrawal ${ }^{2}$, J. N. De ${ }^{1}$ and S. K. Samaddar ${ }^{2}$ \\ (1) Variable Energy Cyclotron Centre, 1/AF, Bidhannagar, Calcutta - 700064, India \\ (2) Saha Institute of Nuclear Physics, 1/AF, Bidhannagar, Calcutta - 700064, India
}

(November 20, 2018)

\begin{abstract}
A detailed microscopic study of the temperature dependence of the shapes of some rare-earth nuclei is made in the relativistic mean field theory. Analyses of the thermal evolution of the single-particle orbitals and their occupancies leading to the collapse of the deformation are presented. The role of the nonlinear $\sigma$-field on the shape transition in different nuclei is also investigated; in its absence the shape transition is found to be sharper.
\end{abstract}

PACS number(s) 21.10.Ma, 21.60.Jz, 27.70.+q 


\section{INTRODUCTION}

Interaction between nucleons in a nucleus may give rise to preferred orientations of the single-particle orbitals leading to deformed intrinsic shapes of the nuclei. With thermal excitations, they undergo a phase transition to spherical shapes. This has been studied in the experiments on the shapes of giant dipole resonances (GDR) built on excited states [1,2]. The understanding of the mean field shape evolution with temperature has been attempted in a macroscopic approach [3, I] generally referred to as the Landau theory of phase transitions. They have also been studied in microscopic framework like finite-temperature non-relativistic Hartree-Fock [5.6] and Hartree-Fock-Bogoliubov (HFB) approaches [7] 9] with the pairingplus-quadrupole $(\mathrm{P}+\mathrm{Q})$ interaction. The shape transition temperature is found to be in the domain of $\sim 1.0$ to $1.8 \mathrm{MeV}$ for the rare-earth nuclei. Here the model Hamiltonian is simplistic, the model space is small, an inert core is assumed and moreover, the role of the Coulomb field is taken into consideration in an effective manner. Recently, we have studied 10,11] this same phenomenon in the framework of relativistic mean field theory (RMF). Here the model space is sufficiently large, all the nucleons are treated on equal footing and the Coulomb interaction is properly accounted for. It is then found that the shape transition temperature is noticeably higher. Except for some calculations in the s-d shell nuclei [12,13], nearly all the calculations have been done for nuclei in the rare-earth region; it is found that the deformation undergoes a sudden collapse at the shape transition temperature. Grossly, one understands the dissolution of the deformation with temperature in these nuclei from the following: shell structure leads to the population of the deformation-driving states, the so-called intruder states producing the static ground state deformation; their depopulation with gradual heating restores the spherical symmetry.

The aim of the present paper is to analyse in more microscopic details the collapse of the deformation with temperature. In doing so, we also explore whether the sudden collapse observed in the rare-earth nuclei is universal or system-specific. The stability towards the deformed ground state for the axially symmetric nuclei that we consider is given by the 
arrangement of the single-particle orbitals of good projection quantum number; the evolution of the energies of these single-particle orbitals with temperature, particularly those near the Fermi surface and also their occupancy evolution lead to a self-consistent rearrangement of the orbitals and thus can reveal in detail why the deformed nuclei undergo a shape transition.

The non-linear $\sigma$-coupling term in the effective Lagrangian has been introduced [14] to reproduce properly the finite size effect, particularly the surface properties of nuclei. Since the spin-orbit coupling term is sensitive to the fall of the density at the nuclear surface, the structure of the Fermi surface is expected to depend on the non-linear term and thus might affect the ground state deformation and its thermal evolution. These aspects are also investigated using a parameter set (HS) where the non-linear $\sigma$-coupling term is absent.

A brief discussion of the theoretical framework we employ is given in section II. The results and discussions are presented in section III. The concluding remarks are given in section IV.

\section{FORMALISM}

The details of the formalism are given in Ref. [10]. For the sake of completeness, however, we write down the effective Lagrangian density describing the nucleon-meson many body system followed by a very brief discussion. The Lagrangian density is given by

$$
\begin{aligned}
\mathcal{L}= & \bar{\Psi}_{i}\left(i \gamma^{\mu} \partial_{\mu}-M\right) \Psi_{i}+\frac{1}{2} \partial^{\mu} \sigma \partial_{\mu} \sigma-U(\sigma)-g_{\sigma} \bar{\Psi}_{i} \sigma \Psi_{i} \\
& -\frac{1}{4} \Omega^{\mu \nu} \Omega_{\mu \nu}+\frac{1}{2} m_{\omega}^{2} \omega^{\mu} \omega_{\mu}-g_{\omega} \bar{\Psi}_{i} \gamma^{\mu} \omega_{\mu} \Psi_{i}-\frac{1}{4} \vec{R}^{\mu \nu} \vec{R}_{\mu \nu}+\frac{1}{2} m_{\rho}^{2} \vec{\rho}^{\mu} \vec{\rho}_{\mu} \\
& -g_{\rho} \bar{\Psi}_{i} \gamma^{\mu} \vec{\rho}_{\mu} \vec{\tau} \Psi_{i}-\frac{1}{4} F^{\mu \nu} F_{\mu \nu}-e \bar{\Psi}_{i} \gamma^{\mu} \frac{\left(1-\tau_{3}\right)}{2} A_{\mu} \Psi_{i} .
\end{aligned}
$$

The mesons included in the description are scalar-isoscalar $\sigma$, vector-isoscalar $\omega$ and vectorisovector $\rho$ mesons. The arrows in Eq.(1) indicate isovector quantities. The scalar selfinteraction term $U(\sigma)$ of the $\sigma$ meson is taken to be non-linear

$$
U(\sigma)=\frac{1}{2} m_{\sigma}^{2} \sigma^{2}+\frac{1}{3} g_{2} \sigma^{3}+\frac{1}{4} g_{3} \sigma^{4} .
$$


The nucleon mass is $M ; m_{\sigma}, m_{\omega}$ and $m_{\rho}$ are the meson masses, $g_{\sigma}, g_{\omega}$ and $g_{\rho}$ are the coupling constants for the mesons and $e^{2} / 4 \pi=1 / 137$ is the fine structure constant. The field tensors for $\omega$ and $\rho$ are given by $\Omega^{\mu \nu}$ and $\vec{R}_{\mu \nu}$; for the electromagnetic field, it is $F^{\mu \nu}$. Recourse to the variational principle followed by the mean field approximation treating the field as $c$-numbers results in the Dirac equation for the nucleon and Klein-Gordon type equations for the mesons and the photon. For the static case, along with the time-reversal invariance and charge conservation, the equations get simplified. The resulting equations, known as the relativistic Hartree equations or RMF equations alongwith the BCS approximation for inclusion of pairing are solved to yield the fields and the single-particle energies.

The self-consistent solutions are obtained using the basis expansion method [15,16]; this yields the quadrupole deformation $\beta_{2}$ and also the single-particle states as a function of temperature.

\section{RESULTS AND DISCUSSIONS}

For the values of the coupling constants and the masses of the mesons and the nucleons occuring in the Lagrangian density given by Eq. (11), we choose the NL3 parameter set. This parameter set reproduces the ground state properties of finite nuclei very well; it yields also the compressional properties satisfactorily [17]. The single-particle states are calculated using spherical oscillator basis with twelve shells. The values of the chemical potential and the pairing gap at a given temperature are determined using all the singleparticle states upto $2 \hbar \omega_{0}$ above the Fermi surface without assuming any core. In order to check the convergence of the calculation, we have enlarged the basis space from twelve to twenty shells and have extended the model space to include single-particle states upto $3 \hbar \omega_{0}$ above the Fermi surface. For this extended model space, the pairing strength is adjusted to reproduce the ground state pairing gap. The change in the values of the observables are found to be insignificant due to this extension of the basis and the model space even at the highest temperature we consider. Continuum corrections are neglected as their effects are 
found to be negligible [11, 18, 19] in the temperature domain of relevance (less than $\sim 3.0$ $\mathrm{MeV}$ ); the shape transition temperatures for the systems considered are within this range.

In the rare-earth region, we have studied the thermal evolution of the shapes of even-even isotopes of $S m, G d$ and $D y$; for neutron number 86 and 88 , their quadrupole deformation $\beta_{2}$ as a function of temperature is presented in Fig. 1. The following aspects are apparent from the figure: (i) for the isotopes differing by two neutrons, the difference in the ground state deformation $\beta_{2}^{0}$ is significant, (ii) addition of two protons does not increase $\beta_{2}^{0}$ significantly (compare $\beta_{2}^{0}$ for ${ }^{148} \mathrm{Sm}$ and ${ }^{150} \mathrm{Gd}$ ), (iii) there is a close correlation between $\beta_{2}^{0}$ and the critical tempeature $T_{c}$ [11] and that (iv) $\beta_{2}$ remains nearly constant with temperature and then there is a sudden collapse of the deformation near $T_{c}$ in a temperature window of $\sim 0.2$ $\mathrm{MeV}$. To elucidate these phenomena more clearly in microscopic terms, we present results for the thermal evolution of the single-particle levels and their occupancies in the next couple of figures for ${ }^{148} \mathrm{Sm}$ and ${ }^{150} \mathrm{Sm}$. These representative cases guide one to draw inference about the shape evolution of the other nuclei. In Figs. 2-5, the single particle levels near the Fermi surface for the protons and the neutrons in ${ }^{148} \mathrm{Sm}$ and ${ }^{150} \mathrm{Sm}$ are displayed as a function of temperature. The Fermi energies are shown by the dashed lines; in the range of the temperature concerned, they are nearly constant. The ground state is axially symmetric and the levels are degenerate in $\pm K, K$ being the projection quantum number. The level structures remain practically unaltered upto $T \sim 0.75 \mathrm{MeV}$ for ${ }^{148} \mathrm{Sm}$ and upto $\sim 1.0 \mathrm{MeV}$ for ${ }^{150} \mathrm{Sm}$ beyond which the different $K$-levels pertaining to a definite $(n l j)$-orbit start to converge. At the temperature $T_{c}\left(1.15 \mathrm{MeV}\right.$ for ${ }^{148} \mathrm{Sm}$ and $1.6 \mathrm{MeV}$ for $\left.{ }^{150} \mathrm{Sm}\right)$ they become degenerate signifying transition to spherical symmetry. It may be mentioned that in the simplistic $(\mathrm{P}+\mathrm{Q})$ model, the phase transition to a spherical shape occurs relatively earlier; in a recent HFB calculation in an extended model space with the realistic Gogny force [20], it has however been found that the transition temperature is in keeping with that found in our calculations in the RMF framework [10].

Examination of the single-particle spectra is helpful in understanding why the two neutron addition to ${ }^{148} \mathrm{Sm}$ increases the ground state deformation whereas the two proton 
addition does not alter it significantly. Because of pairing, the occupancies of the states near the Fermi surface is partial for a nucleus in its ground state. From Figs. 3 and 5 one sees that the two neutron addition increases maximally the population of the ${ }^{1 / 2} f_{7 / 2}$ state (for the orbitals, we have used the notation ${ }^{K} l_{j}$ ); the populations of the states ${ }^{1 / 2} h_{9 / 2}$ and ${ }^{3 / 2} h_{9 / 2}$ are also significantly enhanced. All these orbitals are intrinsically highly prolate which explains the increase in the ground state deformation of ${ }^{150} \mathrm{Sm}$. (Because of axially symmetry in the even-even nuclei we study, the projection $K$ is a good quantum number and is degenerate with $(-K)$ because of time reversal invariance. The states ${ }^{K} l_{j}$ just mentioned are the ones that have the largest amplitude for the orbital with projection $K$ ). From Figs. 2 and 4 we also note that the deep lying ${ }^{7 / 2} g_{7 / 2}$ proton orbital in ${ }^{148} \mathrm{Sm}$ which is intrinsically oblate moves up closer to the Fermi surface in ${ }^{150} \mathrm{Sm}$ and thus is less populated. This also contributes to the increase in the prolate deformation for ${ }^{150} \mathrm{Sm}$. Examination of the single-particle spectra for ${ }^{148} \mathrm{Sm}$ and ${ }^{150} \mathrm{Gd}$ (not shown here) shows that the addition of two protons does not practially affect the neutron occupancies near the Fermi surface; For protons only the ${ }^{3 / 2} h_{11 / 2}$ orbitals get noticeably more populated thus slightly enhancing the deformation in the ground state for the ${ }^{150} \mathrm{Gd}$ nucleus compared to ${ }^{148} \mathrm{Sm}$ as shown in Fig. 1. The ground state deformation properties of $D y$ isotopes can be explained in a similar fashion.

The suddenness of the transition from a prolate to the spherical shape at the temperature $T_{c}$ can be understood as follows: as the temperature rises, the states above the Fermi surface get increasingly more populated at the expense of the states below. This causes a self-consistent reorganization of the single-particle field because of which the single-particle states evolve with temperature as depicted in Figs. 2-5. With increase in temperature for $T$ above $\sim 0.5 \mathrm{MeV}$, one finds from Fig. 6 for the proton orbitals in ${ }^{148} \mathrm{Sm}$ a tendency for equalisation of the single-particle occupancies for different $K$-states emanating from the same $j$-orbital. Around $T_{c}$, one could readily see the sharp drop in the occupancies of the prolate orbitals with an accompanied enhancement of the same for the oblate orbitals resulting in the rapid collapse of the deformation. The neutron orbitals (not shown here) 
have also the similar features. All these arguments apply also for the other rare-earth nuclei considered.

To examine whether the sudden collapse of the deformation as observed in the rare-earth nuclei is a generic feature or system-specific, one needs to consider the shape evolution of some other nuclei in a different mass region. For this purpose, we study two isotopes of zinc nuclei, namely ${ }^{64} Z n$ and ${ }^{66} Z n$. As all the features related to shape evolution in these isotopes are found to be very similar, we discuss only the nucleus ${ }^{64} \mathrm{Zn}$. In Fig. 7, we display the thermal evolution of the deformation $\beta_{2}$ for this nucleus. To draw a comparison of the shape evolution with the rare-earth systems, we also display in the same figure the temperature dependent deformation for the nucleus ${ }^{150} \mathrm{Sm}$. It is seen that the fall-off of the deformation with temperature of ${ }^{64} Z n$ is somewhat slower. This is magnified in Fig.8 where the heat capacity per particle for the two systems ${ }^{150} \mathrm{Sm}$ and ${ }^{64} \mathrm{Zn}$ are displayed. For each curve, the twin peaks at lower temperature correspond to the neutron and proton pairing transitions. For the ligher nucleus, this transition is relatively more prominent and is at a little higher temperature as expected. The peaks at higher temperatures $(\sim 1.6 \mathrm{MeV}$ for $S m$ and $\sim 2.0$ $\mathrm{MeV}$ for $Z n$ ) refer to the shape transition; whereas it is very prominent for the rare-earth system, it is rather weak and looks more like a plateau for the ${ }^{64} \mathrm{Zn}$ nucleus signalling a relatively smoother shape transition to the spherical configuration. It is further that though the ground state deformations of these two nuclei are nearly the same, the transition temperature for ${ }^{64} \mathrm{Zn}$ is significantly higher. Comparison of the thermal evolution of the level structures of ${ }^{64} \mathrm{Zn}$ (shown in Fig.9) with those of ${ }^{150} \mathrm{Sm}$ displayed in Figs.4 and 5 may help in understanding the delayed shape transition in ${ }^{64} \mathrm{Zn}$. For ${ }^{150} \mathrm{Sm}$, in the vicinity of the transition temperature, the single-particle orbitals of opposite intrinsic deformations cross each other near the Fermi surface hastening ite approach towards a spherical configuration. The higher $T_{c}$ in ${ }^{64} \mathrm{Zn}$ may be partially attributed to the absence of these level crossings.

It has been mentioned that the non-linear $\sigma$-coupling term is necessary to explain the surface properties of finite nuclei [14]. Though in the absence of this term, the nuclear matter binding energy and the saturation density are reproduced, the binding energies of finite nuclei 
can not be obtained properly [21]. The fall-off of the nucleon density profile at the surface is found to be stiffer without the non-linear term. Since the spin-orbit splitting is proportional to the density gradient, this splitting is then likely to be larger without the non-linear $\sigma$-term. This may affect the single-particle level structure to which the shape evolution is expected to be sensitive. In order to study this effect on the ground state deformation and the thermal shape evolution of nuclei, calculations have been performed with a parameter set that does not contain the non-linear $\sigma$-coupling term. The HS parameter set [22] is chosen for this purpose. In Fig. 10, the results for the temperature evolution of deformation $\beta_{2}$ are shown for two representative cases, namely, ${ }^{148} \mathrm{Sm}$ and ${ }^{150} \mathrm{Sm}$. For comparison, $\beta_{2}$ with the NL3 parameter set for ${ }^{150} \mathrm{Sm}$ is also displayed in this figure. It is observed that with the HS set the ground state deformation is larger, the transition temperature smaller and that the collapse of the deformation is somewhat faster. The sharper deformation collapse is corroborated in the thermal evolution of the specific heat with and without the non-linear $\sigma$-term in the representative case of ${ }^{150} \mathrm{Sm}$ as shown in Fig. 11 (upper panel). This is amplified further in the case of ${ }^{64} \mathrm{Zn}$ as shown in the bottom panel of the figure. The change in the ground state deformation obtained with the HS parameter set can be understood from the examination of the single-particle spectra near the Fermi surface. For example, as shown in Fig. 12 for the proton single-particle spectra for ${ }^{150} \mathrm{Sm}$, the strong prolate deformationproducing ${ }^{1 / 2} h_{11 / 2}$ and ${ }^{3 / 2} h_{11 / 2}$ orbitals are significantly deeper down the Fermi surface as compared to those displayed in Fig. 4 for the NL3 parameter set. This is mostly responsible for the larger ground state deformation for ${ }^{150} \mathrm{Sm}$. The somewhat sharper dissolution of the deformation with the HS parameter set is again traced back to the self-consistent occupancy evolution with temperature leading to the spherical configuration. Comparison of Fig. 12 with Fig. 4 shows that the spin-orbit splitting is significantly larger (typically $\sim 40 \%$ ) in case of HS parameter set as envisaged earlier. 


\section{CONCLUSIONS}

In this paper, we have attempted to understand in microscopic detail the shape evolutions of deformed nuclei with temperature in the RMF framework. For the rare-earth nuclei we have studied, namely, $S m, G d$ and $D y$ with 86 and 88 neutrons, it is seen that the ground state deformation increases with addition of neutrons appreciably whereas it is not that sensitive to proton addition. Similar inference on the sensitivity of the shape transition temperature can be drawn to neutron and proton addition. It has been found that these transition temperatures are noticeably larger compared to those obtained in the schematic model with pairing-plus-quadrupole interaction but agree reasonably well with those calculated 20] using a realistic force like the Gogny force. From microscopic viewpoint the ground state deformation can be understood in terms of the single-particle level structure near the Fermi surface. The sudden drop of the deformation can also be understood from the temperature-driven fast equalisation of the occupancies of different $K$-states originating from the same $j$-orbital near the Fermi surface close to the transition temperature.

We have also investigated the role of the non-linear self-coupling of the $\sigma$ mesons on the shape evolution of the nuclei. In absence of the non-linear term, the spin-orbit splitting is

enhanced which affects the single-particle level structure near the Fermi surface leading to a sharper shape transition at a lower temperature. 


\section{REFERENCES}

[1] K. A. Snover, Annu. Rev. Nucl. Part. Sci. 36, 545 (1986).

[2] J. J. Gaardhoje, Annu. Rev. Nucl. Part. Sci. 42, 483 (1992).

[3] S. Levit and Y. Alhassid, Nucl. Phys. A413, 439 (1984).

[4] Y. Alhassid, J. Zingman and S. Levit, Nucl. Phys. A469, 205 (1987).

[5] M. Brack and P. Quentin, Phys. Scr. A10, 163 (1974).

[6] P. Quentin and H. Flocard, Annu. Rev. Nucl. Part. Sci. 28, 523 (1978).

[7] A. L. Goodman, Phys. Rev. C34, 1942 (1986).

[8] A. L. Goodman, Phys. Rev. C38, 977 (1988).

[9] J. L. Egido, P. Ring and H. J. Mang, Nucl. Phys. A451, 77 (1986).

[10] B. K. Agrawal, Tapas Sil, J. N. De and S. K. Samaddar, Phys. Rev. C62, 044307 (2000).

[11] B. K. Agrawal, Tapas Sil, S. K. Samaddar and J. N. De, Phys. Rev. C (in press).

[12] H. G. Miller, R. M. Quick, G. Bozzolo and J. P. Vary, Phys. Lett. B168, 13 (1986).

[13] H. G. Miller, R. M. Quick and B. J. Cole, Phys. Rev. C39, 1599 (1989).

[14] J. Boguta and A. R. Bodmer, Nucl. Phys. A292, 413 (1977).

[15] Y. K. Gambhir, P. Ring and A. Thimet, Ann. Phys. (N.Y.) 198, 132 (1990).

[16] P. Ring, Y. K. Gambhir and G. A. Lalazissis, Comp. Phys. Comm. 105, 77 (1997).

[17] G. A. Lalazissis, J. König and P. Ring, Phys. Rev. C55, 540 (1997).

[18] B. K. Agrawal, S. K. Samaddar, J.N.De and S. Shlomo, Phys. Rev. C58, 3004 (1998).

[19] P. Bonche, S. Levit and D. Vautherin, Nucl. Phys. A436, 265 (1985).

[20] J. L. Egido, L. M. Robledo and V. Martin, Phys. Rev. Lett. 85, 26 (2000). 
[21] B. D. Serot and J. D. Walecka, Adv. Nucl. Phys. 16, 1 (1986).

[22] C. J. Horowitz and B.D. Serot, Nucl. Phys. A368, 503 (1981). 


\section{Figure Captions:}

Fig. 1: The temperature evolution of the deformation $\left(\beta_{2}\right)$ for a few rare-earth nuclei with the NL3 parameter set.

Fig. 2: The proton single-particle level spectrum near the Fermi surface for ${ }^{148} \mathrm{Sm}$ as a function of temperature with the NL3 parameter set. The dashed line corresponds to the Fermi surface.

Fig. 3: The neutron single-particle level spectrum near the Fermi surface for ${ }^{148} \mathrm{Sm}$ as a function of temperature with the NL3 parameter set. The dashed line corresponds to the Fermi surface.

Fig. 4: Same as Fig. 2 for ${ }^{150} \mathrm{Sm}$.

Fig. 5: Same as Fig. 3 for ${ }^{150} \mathrm{Sm}$.

Fig. 6: The thermal evolution of single-particle occupancy for protons in ${ }^{148} \mathrm{Sm}$ for a few levels with good projection quantum number $K$ (as indicated) near the Fermi surface. The degeneracy of $\pm K$ has been taken into account in the occupancy.

Fig. 7: Comparison of the thermal evolution of deformation $\beta_{2}$ for ${ }^{64} \mathrm{Zn}$ (solid line) with ${ }^{150} \mathrm{Sm}$ (dotted line).

Fig. 8: The specific heat per particle as a function of temperature for the systems ${ }^{150} \mathrm{Sm}$ and ${ }^{64} Z n$.

Fig. 9: The proton (top panel) and neutron (bottom panel) single-particle spectra for ${ }^{64} \mathrm{Zn}$ near the Fermi surface as a function of temperature.

Fig. 10: The thermal evolution of deformation for ${ }^{148} \mathrm{Sm}$ and ${ }^{150} \mathrm{Sm}$ with the HS parameter set. For the sake of comparison, the same for ${ }^{150} \mathrm{Sm}$ with the NL3 parameter set is also shown. 
Fig. 11: The specific heat per particle as a function of temperature for ${ }^{150} \mathrm{Sm}$ with the HS (solid line) and the NL3 (dotted line) parameter sets (top panel). In the bottom panel, the same is shown for ${ }^{64} Z n$.

Fig. 12: The proton single-particle level spectrum near the Fermi surface with the HS parameter set for ${ }^{150} \mathrm{Sm}$ as a function of temperature. 


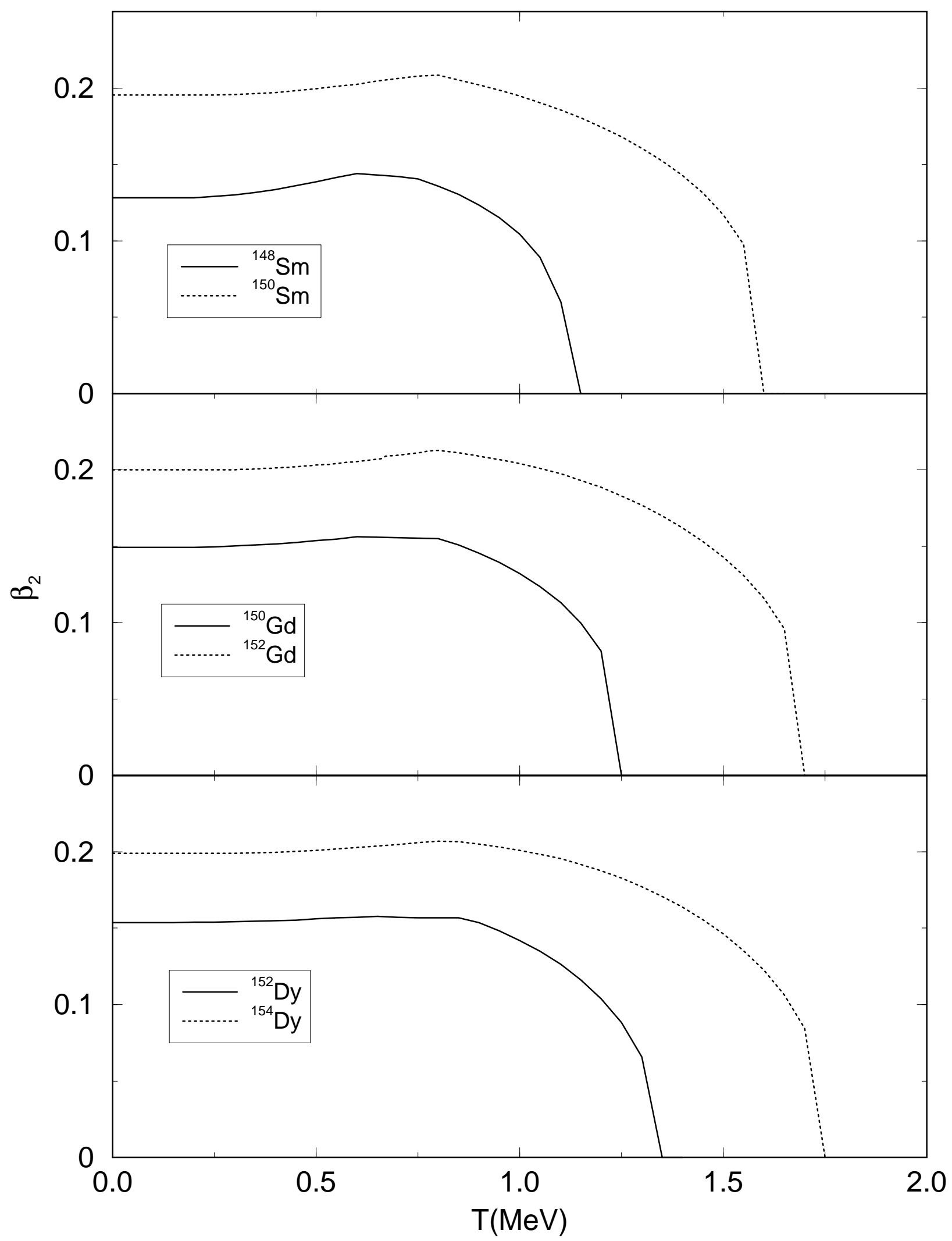

Fig. 1 


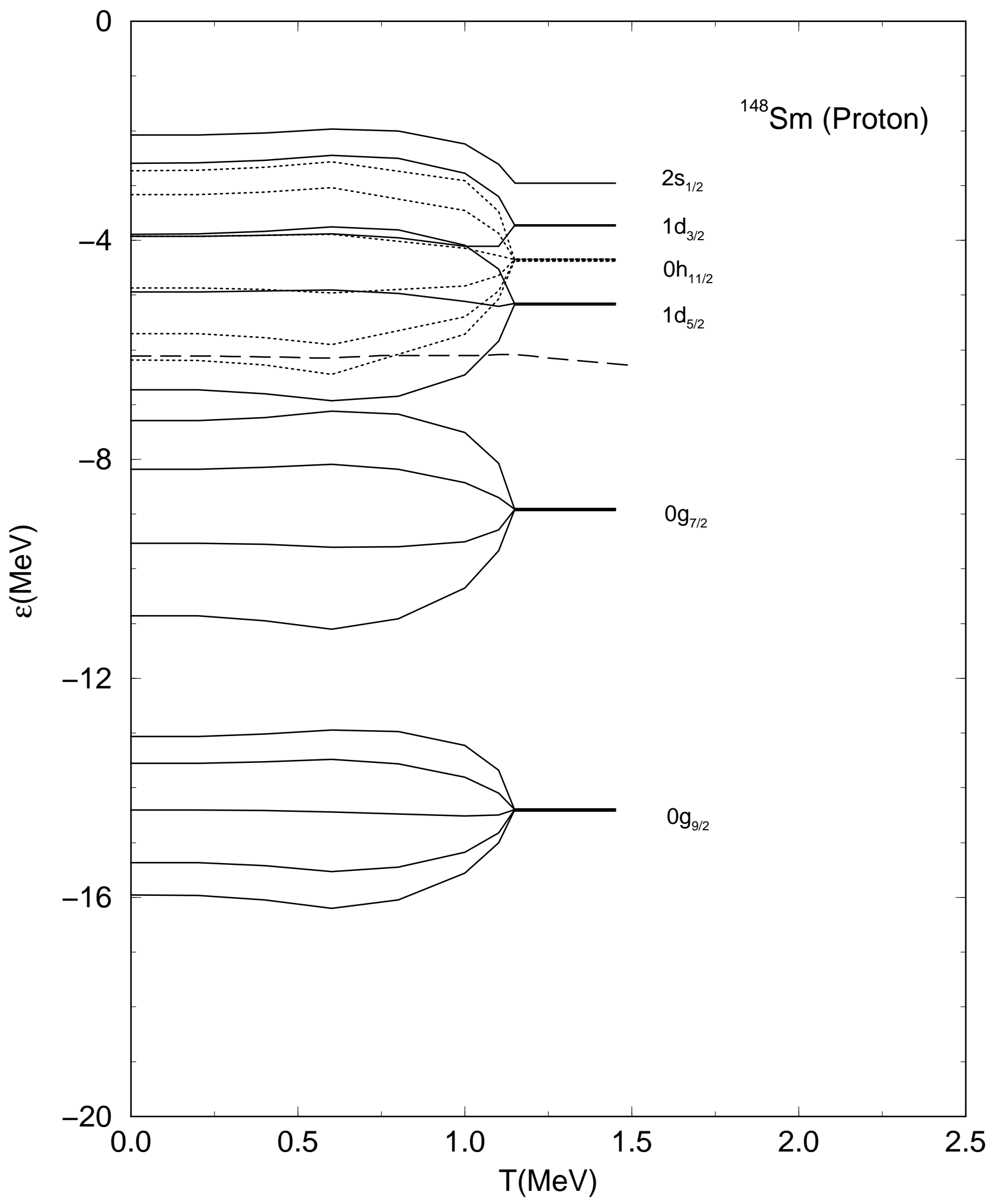

Fig. 2 


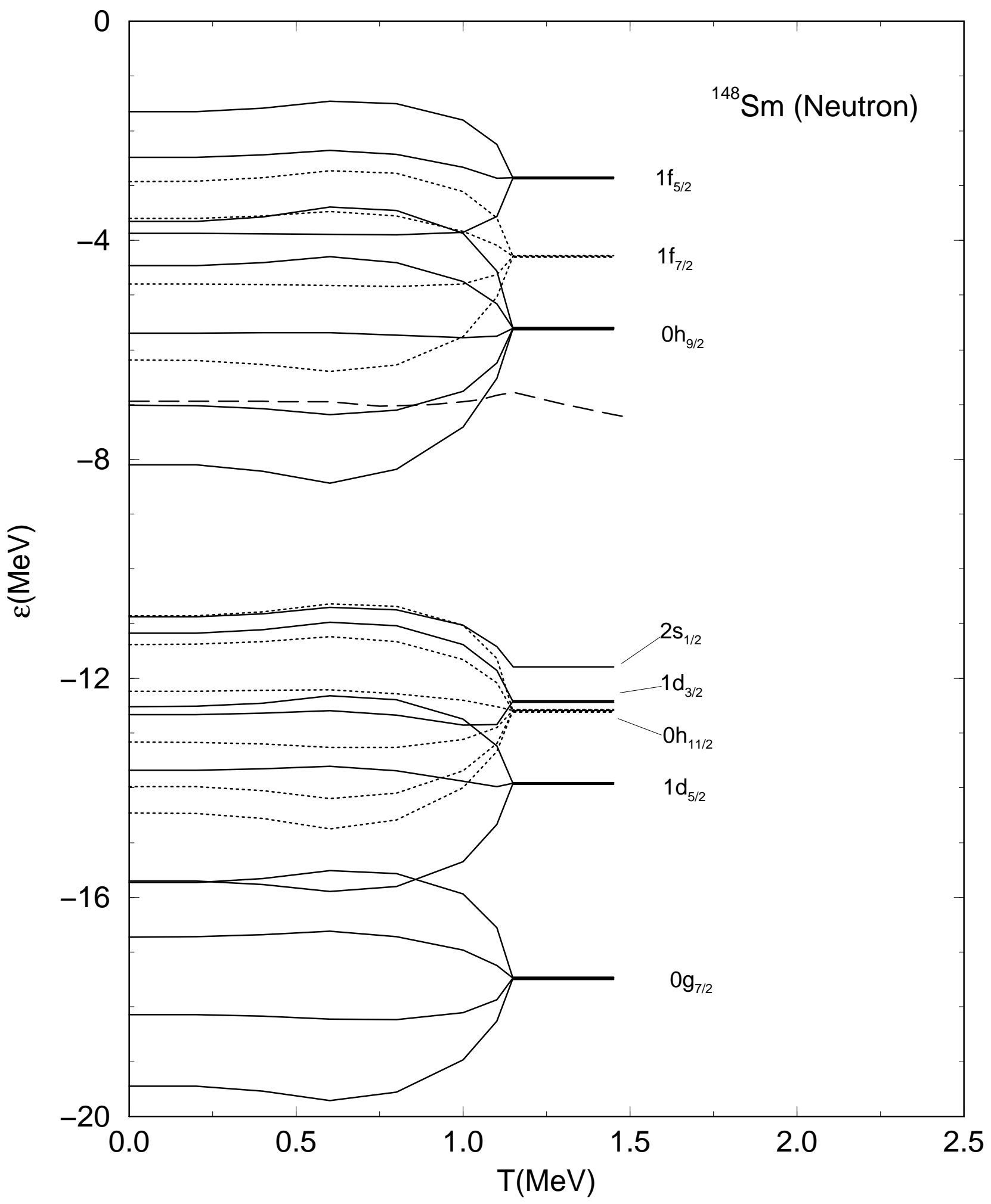

Fig. 3 


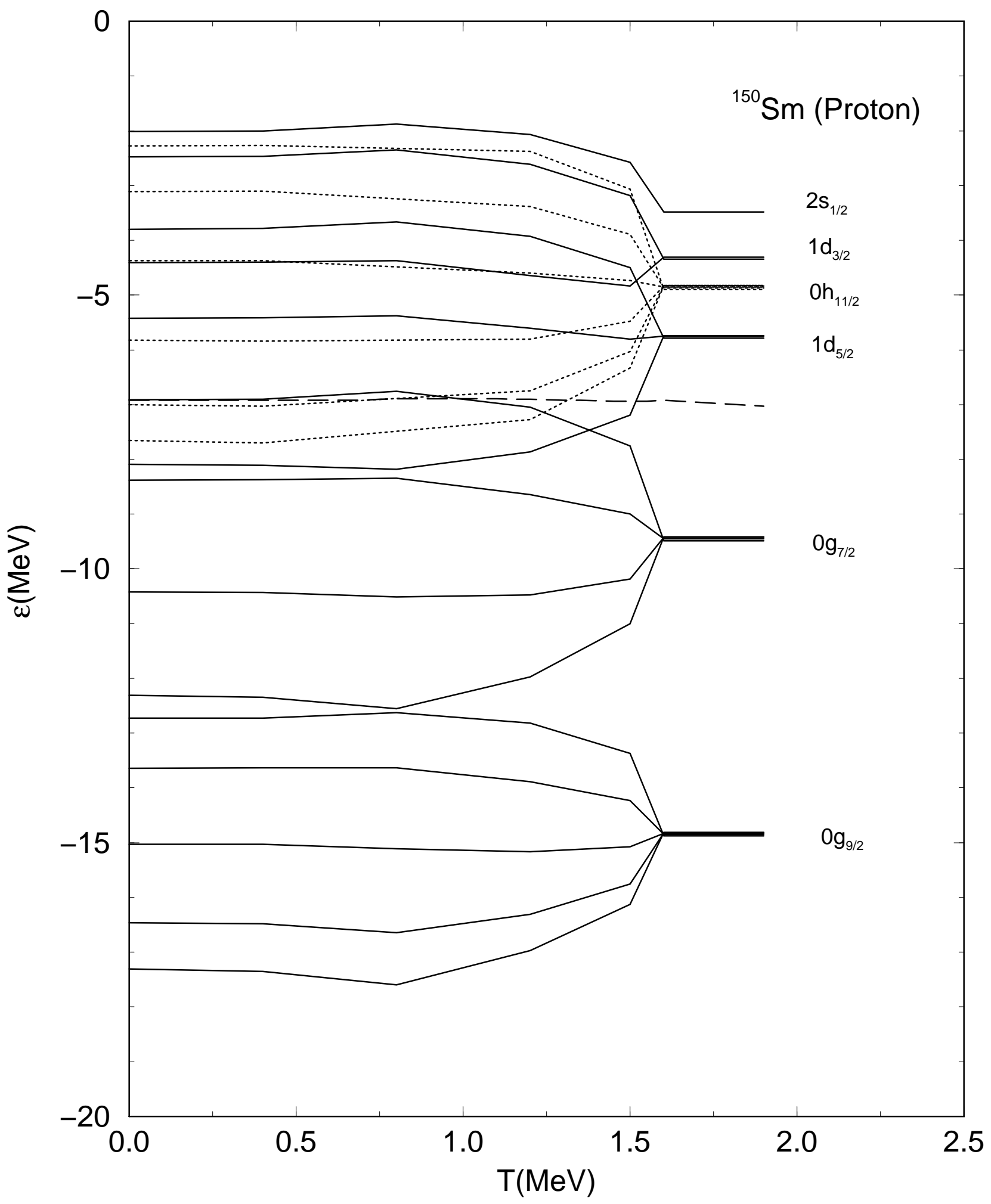

Fig. 4 


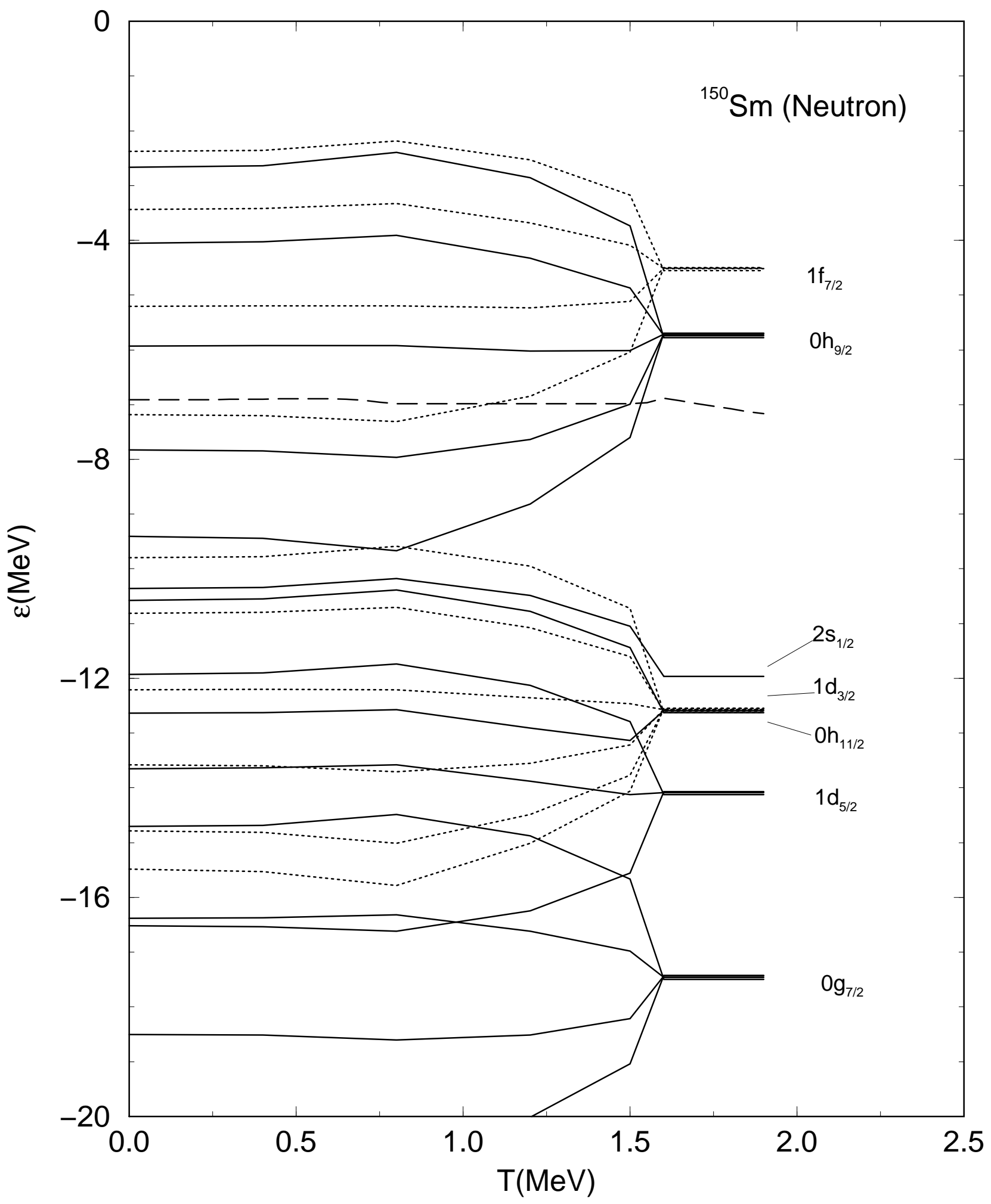

Fig. 5 


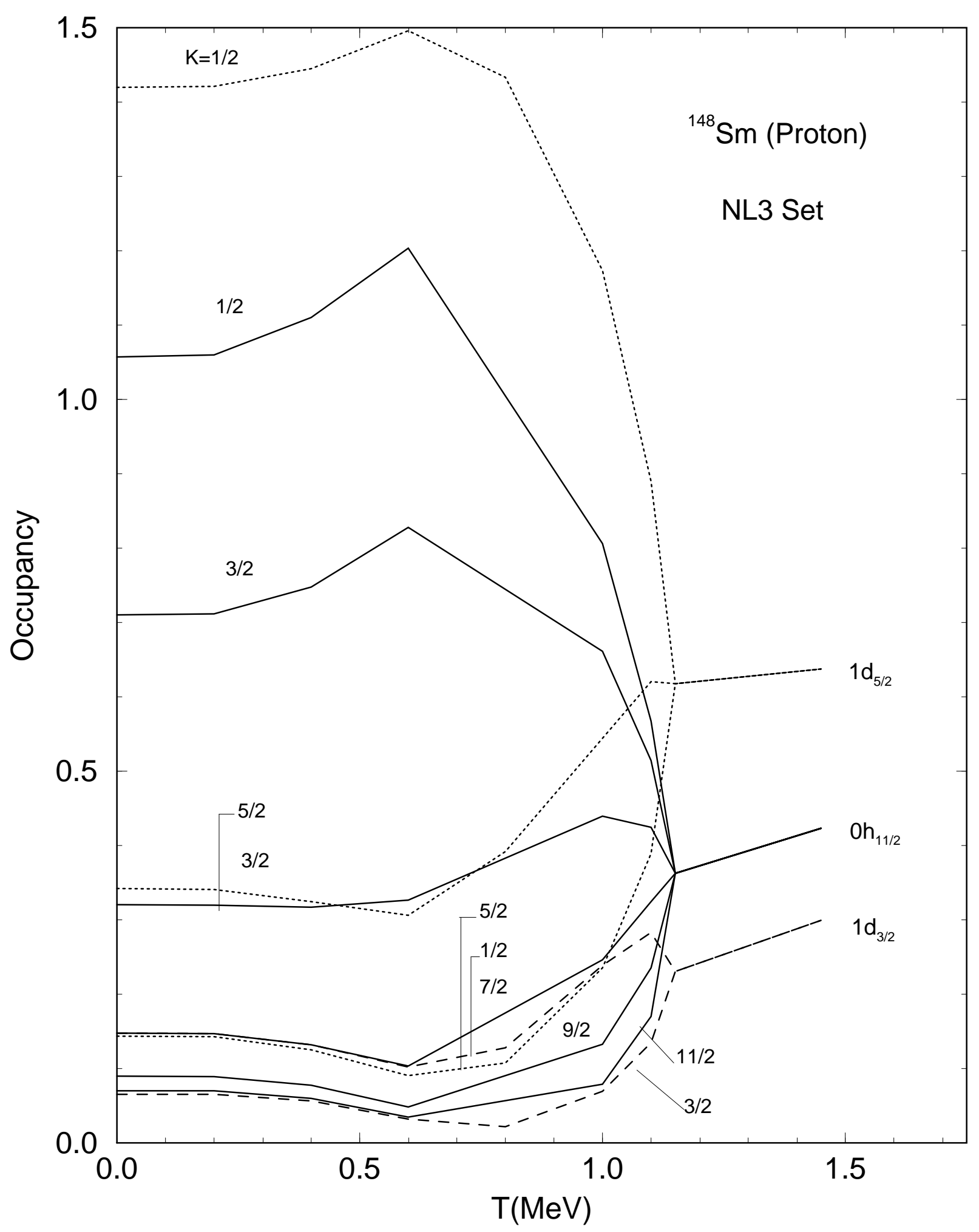

Fig. 6 


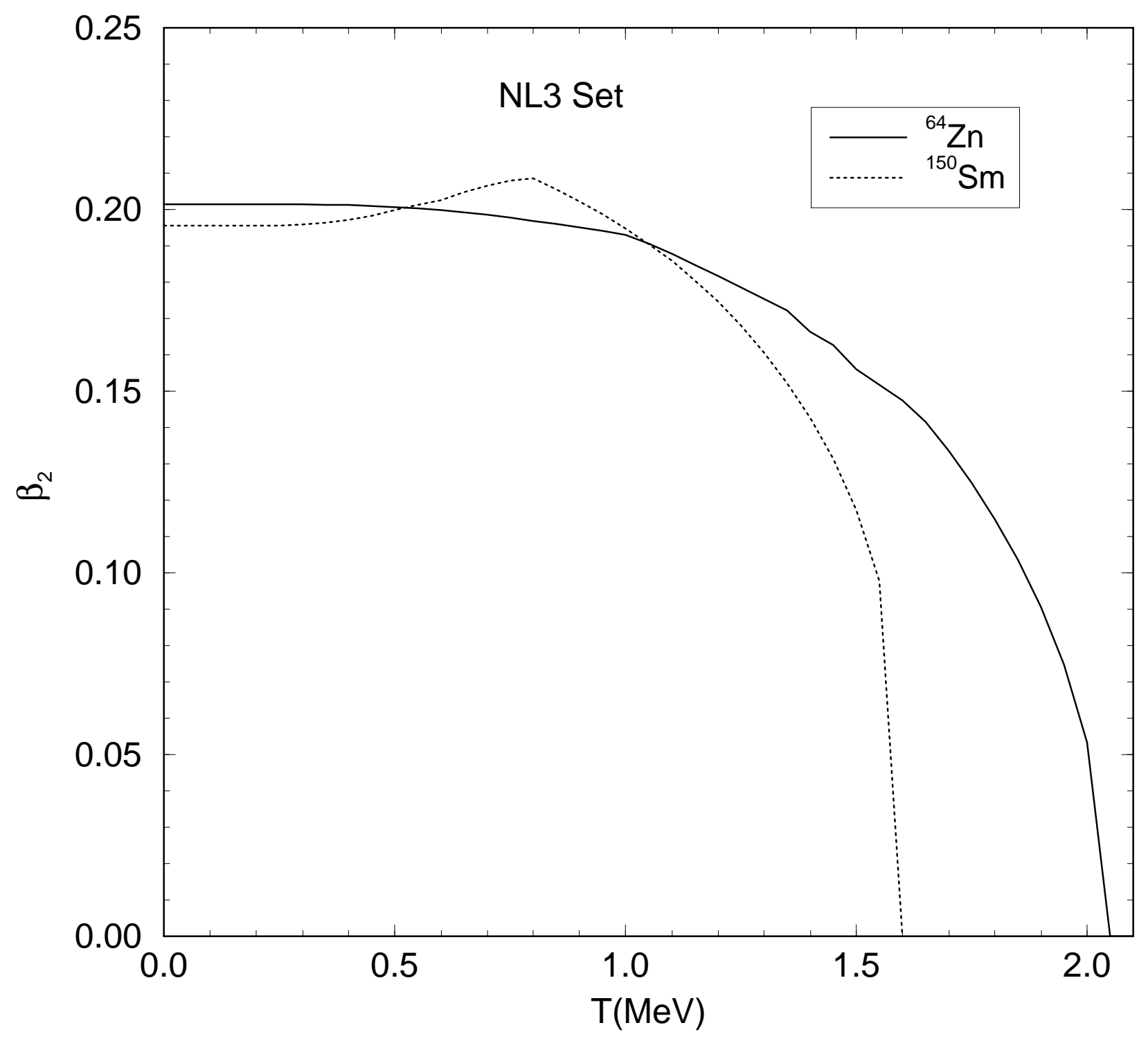

Fig. 7 


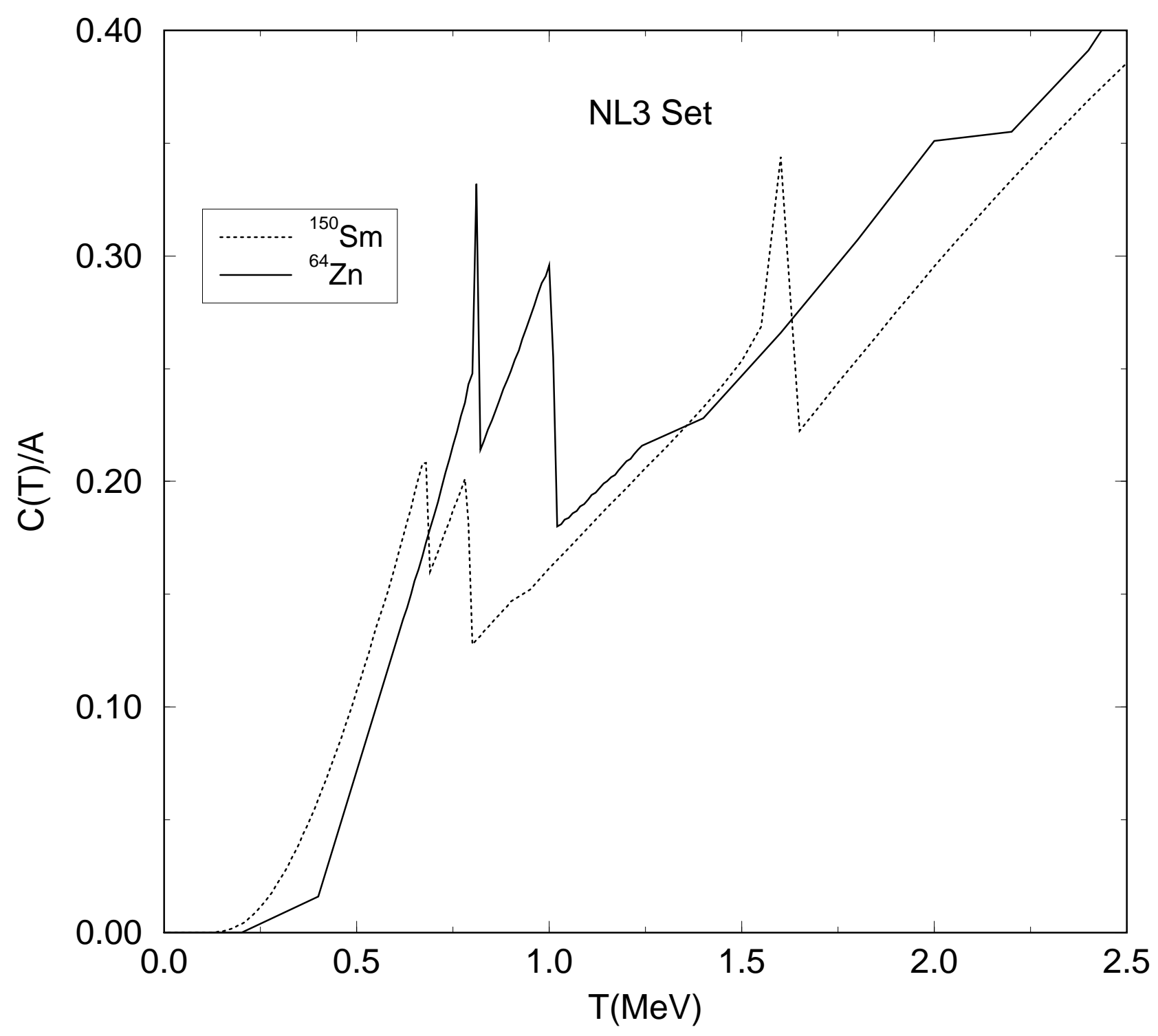

Fig. 8 


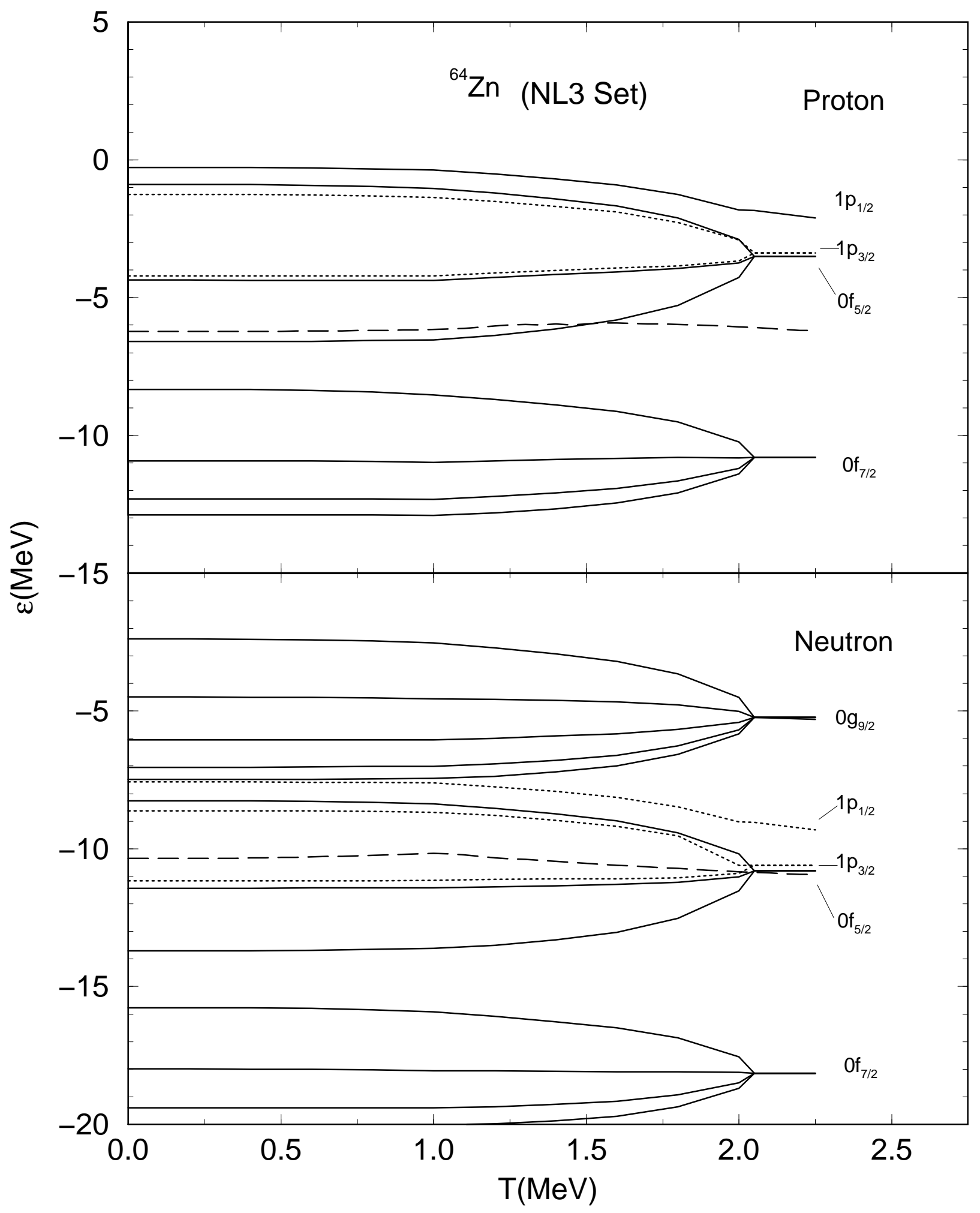

Fig. 9 


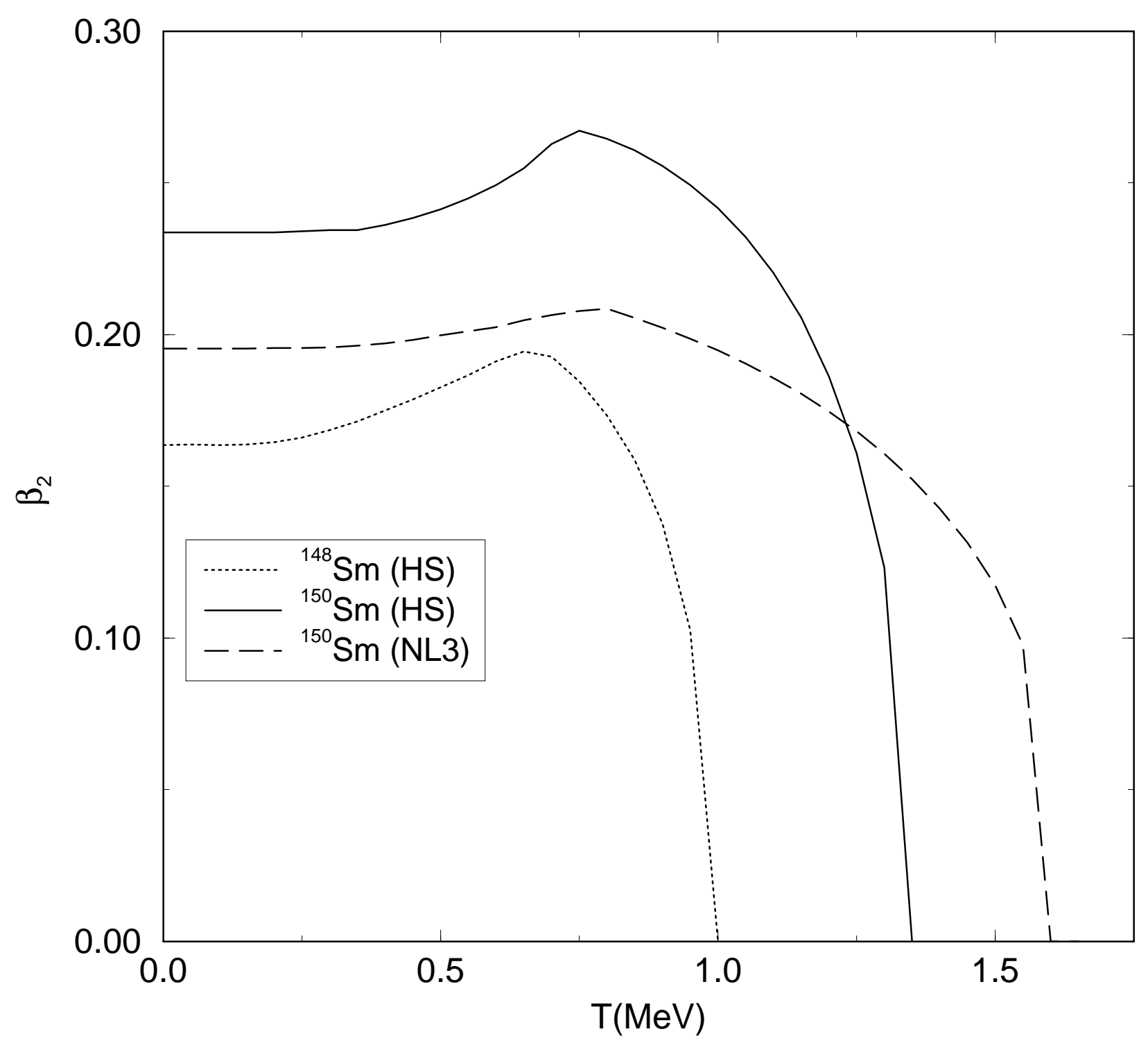

Fig. 10 


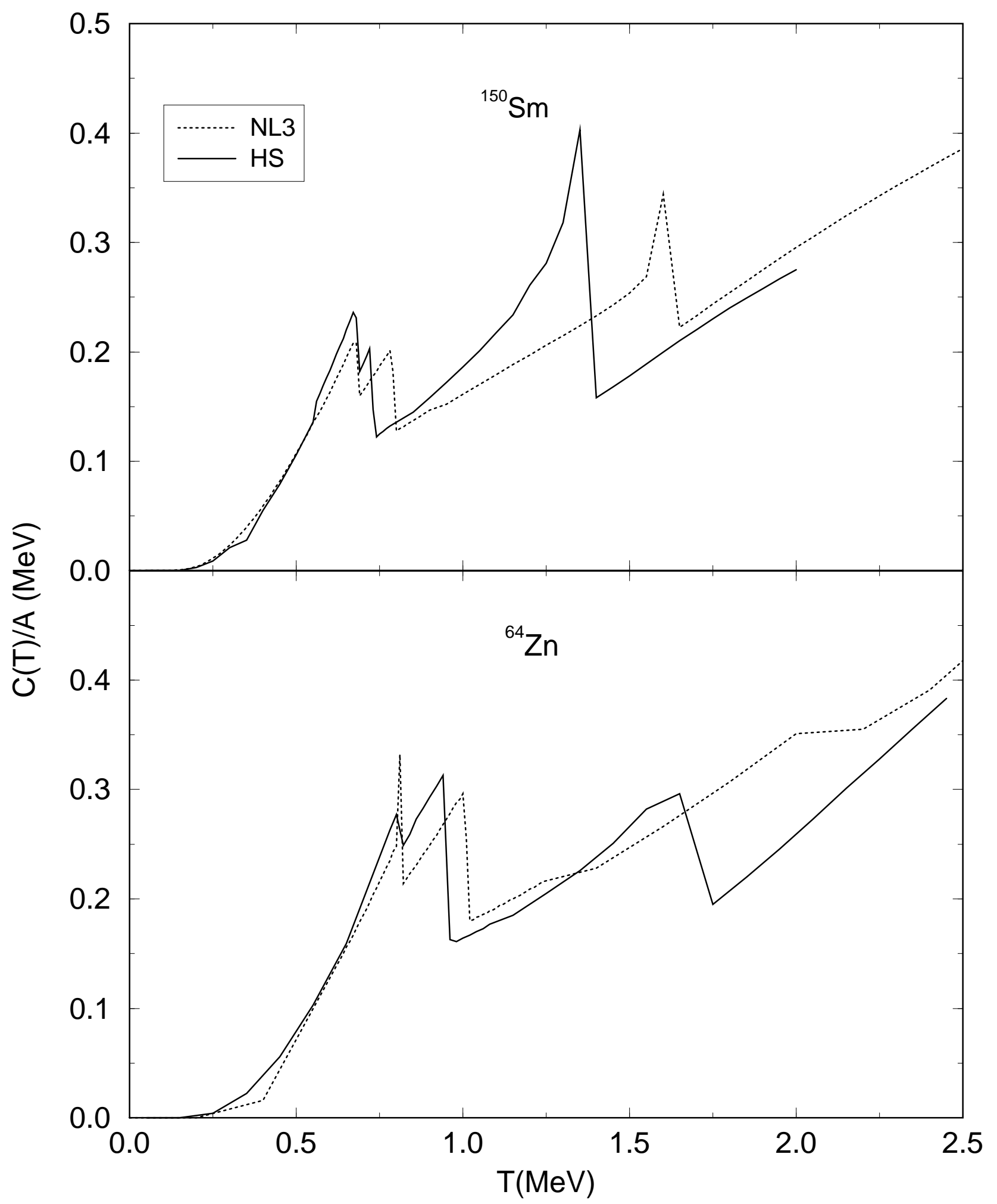

Fig. 11 


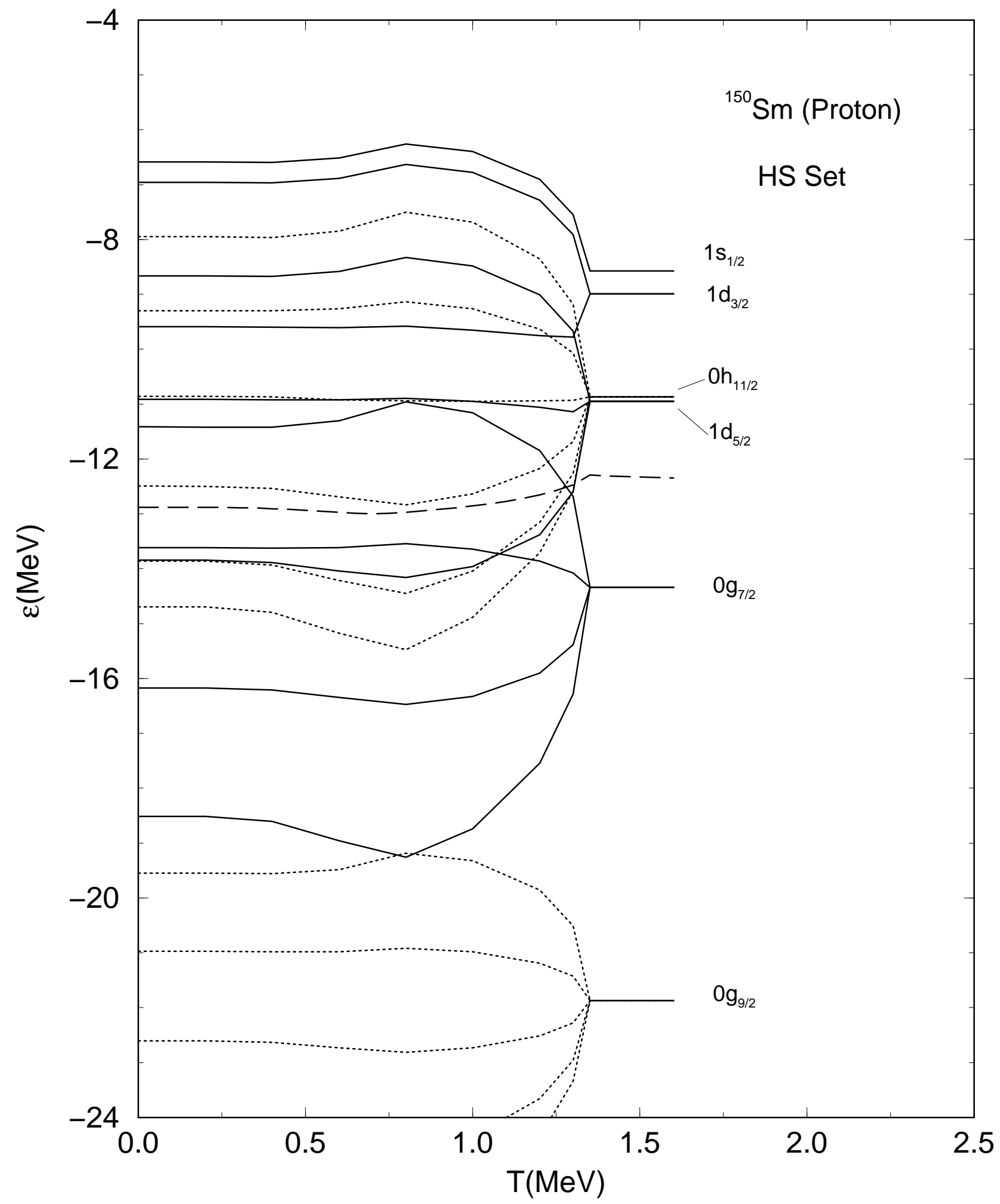

Fig. 12 